Rev. Elev. Méd. vét. Pays trop., 1979, 32 (1) : $53-55$

\title{
Note sur la dicrocoeliose à Dicrocoelium hospes (Looss, 1907) au Niger
}

\author{
par P. TAGER-KAGAN \\ (avec la collaboration technique de DJIBO GARBA (*) et NAINOU GUERO (*))
}

\section{RÉSUMÉ}

A la suite de la découverte de Dicrocoelium hospes chez des zébus lors d'un essai de médicaments, on a recherchè durant un an sur trois marchés du département de Niamey les animaux porteurs de ce parasite (bovins, ovins, caprins). L'aire de répartition de ce trématode peut être étendue jusqu'au $14^{\text {e }}$ parallèle de latitude nord.

\section{INTRODUCTION}

A notre connaissance, aucune note dans la littérature vétérinaire du Niger ne parle de la dicrocoeliose à Dicrocoelium hospes (Loos, 1907) ; ce trématode est responsable d'une distomatose hépatique par sa pénétration dans le parenchyme hépatique puis dans les canaux biliaires des ruminants domestiques.

On peut trouver des références concernant ce parasite dans les pays limitrophes du Niger et en Afrique de l'Ouest dans les travaux de GRABER et OUMATIE (4) pour le Tchad et le Cameroun, LAPAGE (6) et SCHILLHORN VAN VEEN (T. W.), SHONEKAN (R. A. O.) FABIYI (J. P.) (9) pour le Nigeria, BOURGAT et SEGUIN (I.) pour le Togo, ODEI (8) et FISCHTHAL (J. H.), THOMAS (J. D.) (3) pour le Ghana, WILLIAMS (M. O.) (11) pour la Sierra Leone.

En juillet 1974, lors d'un essai de traitement du polyparasitisme du zébu à l'aide d'associations médicamenteuses (5) nous avons mis Dicrocoelium hospes en évidence pour la première fois sur 4 bovins ( 75 animaux avaient été autopsiés) ; 2 zébus étaient porteurs de plusieurs milliers de

(*) Laboratoire de l'Elevage, INRAN, B. P. 485, Niamey (Rép. du Niger). parasites, les 2 autres présentaient une pauci infestation (189 et 973 Dicrocoelium).

A la suite de cette découverte, nous avons décidé, lors de nos tournées hebdomadaires sur différents marchés du département de Niamey, de rechercher systématiquement sur les animaux abattus la présence de Dicrocoelium hospes.

\section{MATÉRIEL ET MÉTHODE}

Trois marchés ont été choisis (voir carte) :

- Torodi ;

- Gotheye ;

- Tillabery.

Une fois par semaine, les animaux (bovins, ovins et caprins) de ces marchés, abattus pour la boucherie, ont eu leur foie et vésicule biliaire soigneusement examinés afin de mettre en évidence la présence ou non de Dicrocoelium hospes; de plus, un certain nombre d'exemplaires ont été examinés après coloration au carmin chlorhydrique: il s'agissait bien de Dicrocoelium hospes tel quel'ont décrit GRABER et collab. (4), BOURGAT et collab. (1). Nous avons ainsi examiné sur l'ensemble des 3 marchés :

- 394 bovins ;

- 890 ovins ;

- 3045 caprins. 


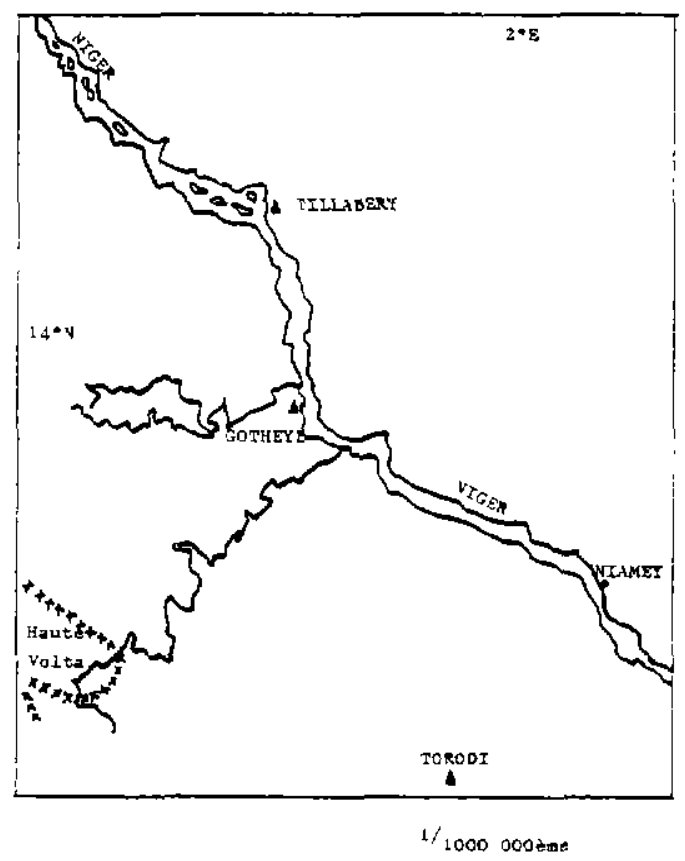

- Localisation des enquetes

\section{RÉSULTATS}

\section{Taux et niveau d'infestation}

Nous avons groupé les résultats de nos observations dans le tableau $\mathbf{I}$.

Un foyer important existe dans la région de TORODI ( $13^{\circ} 7$ de latitude nord); les zébus sont les plus atteints; 94 p. 100 des bovins étaient porteurs de Dicrocoelium hospes contre 27 p. 100 et 26 p. $100 \mathrm{chez}$ les ovins et caprins. Ce foyer n'est pas spécifiquement nigérien car le marché de Torodi est alimenté par de nombreuses bêtes venues de Haute-Volta.

A Gotheye (1350 de latitude nord) situé sur la rive droite du fleuve Niger, le pourcentage des animaux atteints est moins important:
54 p. 100 des bovins, 14 p. 100 des ovins et 15 p. 100 des caprins sont infestés par la petite douve. Nous avons observé la coexistence de Fasciola gigantica et de Dicrocoelium hospes sur 26 p. 100 des bovins.

Sur le marché de Tillabery $\left(14^{\circ} 13\right.$ de latitude nord), situé sur la rive gauche du fleuve Niger, les infestations sont discrètes; aucun bovin n'était porteur de Dicrocoelium, 2 p. 100 des ovins et 10 p. 100 des caprins étaient parasités par ce trématode.

\section{Variations saisonnières}

Chez les bovins, l'infestation sévit toute l'année, mais on observe des variations importantes selon l'origine géographique des animaux; ainsi, à Torodi, le taux d'infestation dépasse 85 p. 100 toute l'année sauf en août ( 80 p. 100$)$. A Gotheye, l'infestation est minimale (35 p. 100) en février-mars, puis elle remonte progressivement pour atteindre un maximum en juillet (77 p. 100).

Chez les ovins, le nombre d'animaux infestés est :

- à Torodi, supérieur à 30 p. 100 en décembre-janvier et en mars-avril, inférieur à 30 p. 100 le reste de l'année ;

- à Gotheye, voisin de 30 p. 100 en mars et en septembre, compris entre 6 et 15 p. 100 le reste de l'année ;

- à Tillabery, la dicrocceliose n'existe pas de septembre à janvier. Elle sévit à un faible taux (3 à 6 p. 100) le reste de l'année.

Chez les caprins, les minimums (20 p. 100$)$ se situent de mai à juillet et en août à Torodi, en juillet, août et septembre $(10$ p. 100) à Gotheye, en mars-avril et en août-septembre à Tillabery. Le reste du temps, le taux d'infestation oscille entre 10 et 40 p. 100.

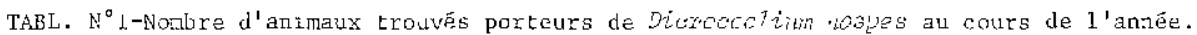

\begin{tabular}{|c|c|c|c|c|c|c|c|c|c|}
\hline & \multicolumn{3}{|c|}{$B \circ v 1 \pi s$} & \multicolumn{3}{|c|}{$0 \mathrm{v} I \mathrm{~ns}$} & \multicolumn{3}{|c|}{$\mathrm{caprins}$} \\
\hline & $\begin{array}{l}\text { Antzalux } \\
\text { examines }\end{array}$ & $\begin{array}{l}\text { Animaux } \\
\text { parasités }\end{array}$ & 1.700 & $\begin{array}{r}\text { Mnimaux } \\
\text { exam1nés }\end{array}$ & $\begin{array}{c}\text { Animaux } \\
\text { parasitês }\end{array}$ & p. 100 & $\begin{array}{r}\text { Anımaux } \\
\text { examinés }\end{array}$ & $\begin{array}{c}\text { Animaux } \\
\text { parasités }\end{array}$ & p. 100 \\
\hline T'orodi & 211 & 200 & 94 & 330 & 92 & 27 & 1435 & 376 & 26 \\
\hline Gotheye & 183 & 100 & 54 & 303 & 44 & 14 & I 169 & 180 & 15 \\
\hline Tillabery & - & - & - & 257 & 6 & 2 & 441 & 46 & 10 \\
\hline Total & 394 & 300 & 76 & 890 & 142 & I6 & 3045 & 602 & 19 \\
\hline
\end{tabular}


En définitive, chez les bovins la dicrocœliose — là où elle existe - se voit toute l'année avec des minimums relatifs en saison sèche (févriermars) et au milieu de la saison des pluies (août). Chez les ovins et les caprins, les minimums ont tendance à se situer en saison des pluies et cela est vraisemblablement lié à la biologie propre des seconds hôtes intermédiaires, c'est-à-dire des fourmis. SEGUIN, au Togo, a observé à peu près le même phénomène.

\section{CONCLUSION}

Les enquêtes menées durant une année dans le département de Niamey à la suite de la mise en évidence de Dicrocoelium hospes sur des zébus ont montré que l'aire de répartition de ce parasite est reportée jusqu'au $14^{\text {e }}$ parallèle de latitude nord, alors que l'on estimait jusqu'à présent, que la limite nord de Dicrocoelium hospes était le $11^{\mathrm{e}}$ parallèle.

\section{SUMMARY}

\section{Note about Dicrocoeliosis (Dicrocoetium hospes (Looss, 1907)) in Niger)}

Following discovery of Dicrocoelium hospes (Looss, 1907) on zebu at the time of a test of drugs, we have searched during one year in three markets of the department of Niamey the animals carrying this parasite (bovine, ovine, goat).

Area of repartition of Dicrocoelium hospes can be extented up to the 14th parallel of north latitude.

\section{RESUMEN}

\section{Nota sobre la dicroceliosis (Dicrocoelium hospes (Looss, 1907)) en el Niger}

Luego de descubrir Dicrocoelium hospes en cebus durante un ensayo de medicamentos, se han buscado durante un año los animales parasitados (bovinos, ovinos, cabrunos) en tres mercados del departamento de Niamey.

Elárea de repartición de dicho Tremátodo ịpuede extenderse hasta el paralelo catorce latitud Norte.

\section{BIBLIOGRAPHIE}

1. BOURGAT (R.), SEGUIN (D.), BAYSSADEDUFOURC. Données nouvelles sur Dicrocoelium hospes (Looss, 1907). Anatomie de l'adulte et cycle évolutif. Annls Parasit. hum. comp., 1975, 50 (6) : 701-714.

2. EUZEBY (J.). Maladies vermineuses des anımaux domestiques. Tome II, deuxième fascicule, livre I. Distomatoses hépato-biliaires. Paris, Vigot Frères.

3. FISCHTHAL (J. H.), THOMAS (J. D.). Two dicrocoelisd trematodes from Ghana: Concinnum ghanensis and Dicrocoelium hospes (Looss, 1907) J. Helminth., 1971, 45 (4) : 337-340.

4. GRABER (M.), OUMATIE (O.). Existence en Afrique équatoriale d'un important foyer de dicrocoellose bovine et ovine à Dicrocoelium hospes (Looss, 1907). Rev. Elev. Méd. vét. Pays trop., 1964, 17 (4): 532-533.

5. GRABER (M.), TAGER-KAGAN (P.). Essai de traitement du polyparasitisme du zébu à l'aide des associations Disto 5-Thiabendazole et Disto 5 . Tartrate de Morantel. Rapport. Maisons-Alfort, I. E. M. V. T., 1974.
6. LAPAGE (G.). Monnig's veterinary helminthology and entomology, 5th ed. London, Bailliere Tindall and Cassel, 1965.

7. LUCIUS (R.), FRANK (W.). Beltrag zur Biologle von Dicrocoelium hospes (Looss, 1907). (Trématodes, Dicrocoeliidae). Acta trop., 1978, 35 (2) : 164-181.

8. ODEI (M. A.). A note on dicrocoeliasis and F. giganisca infection in livestock in Northern-Ghana. Ann. trop. Med. Parasit., 1966, $60: 215$.

9. SCHILLHORN VAN VEEN (T. W.), SHONEKAN (R. A. O.), FABIYI (J. P.). A host parasite checklist of helminth parasites of domestic animals in Northern Nigeria. Bull. anim. Hlth Prod. Afr., 1975, 23 (3) : 269-283.

10. SEGUIN (D.). Contribution à l'étude des distomatoses hépato-biliaıres des ruminants au Togo. Thèse Doct. vét. Lyon. 1975, no 64 .

11. WILLIAMS (M. C.). First record of dicrocoeliasis in cattle in Sierra-Leone. Bull. epizoot. Dis. Afr., $1969,17: 433-434$. 